\title{
Outcomes in Patients with Ischemic Cerebrovascular Disease
}

\author{
AKIRA ISHIBASHI AND YOSHITAKE YOKOKURA \\ Departments of Neurosurgery and Surgery, Yokokura Hospital, \\ Miike-gun 839-0295, Japan
}

\begin{abstract}
Summary: An analysis was conducted to determine at discharge the outcomes associated with risk factors in 246 patients with ischemic cerebrovascular disease who were admitted within 48 hours after the onset of the disease. Statistical analysis in this study disclosed that atrial fibrillation was a significant risk factor contributing to the worse outcomes among the subtypes of the ischemic cerebrovascular disease.
\end{abstract}

Key words outcome, cerebral ischemia, atrial fibrillation

\section{INTRODUCTION}

To prevent ischemic cerebrovascular disease, a variety of researches on risk factors associated with ischemic cerebrovascular disease have been done and documented that hypertension (HT), hyperlipidemia (HLP), diabetes mellitus (DM) and atrial fibrillatin (AF) are the major risk factors regarding to the occurrence of ischemic cerebrovascular disease [15]. The purpose of the present study was to determine at discharge the outcomes in patients with ischemic cerebrovascular disease in association with risk factors, such as HT, HLP, DM, AF, and the subtypes of the disease. Age and gender were also analyzed in the study.

\section{PATIENTS AND METHODS}

Between January 1998 and June 2000, consecutive 246 patients with ischemic cerebrovascular disease were admitted to our institution within 48 hours after the onset. On admission, their neurological status and neuroradiology were assessed and as being defined by the World Heath Organization [6], the patients were classified in the following subtypes: lacunar infarction (LI), atherothrombotic infarction (ATI), cardioembolic infarction (CEI), and transient ischemic attack (TIA). In regard to the risk factors associated with the outcomes in each subtype of the disease, HT, HLP, AF, and DM, as well as age and gender were studied using the multiple logistic regression analysis and the analysis adjusted for age and gender. The Glasgow Outcome Scores at discharge were noted [7]. Excellent outcomes describe no symptoms; good outcomes indicate no disability despite some symptoms, or have slight disability in previous activity; moderate disability needs some help walking; severe disability requires nursing care; worse outcomes are vegetative and death.

\section{RESULTS}

This study included 117 males and 129 females and the mean age for the patients upon diagnosis was 74.8 years (range 64.2 to 85.5 years). The number of patients in each subtype of the disease was the following: LI ( $\mathrm{n}=92)(37 \%)$, ATI $(\mathrm{n}=49)(19.9 \%)$, CEI $(\mathrm{n}=54)(21.6 \%)$, TIA $(\mathrm{n}=48)(19.5 \%)$, and unclassified $(\mathrm{n}=3)(0.01 \%)$ (Fig. 1).

One hundred and five (44\%) of 239 patients had HT; and 40 (17.8\%) of 225 patients showed DM; 35 (15\%) of 233 patients had HLP. HT, followed by HLP, and DM, was found to be a higher risk factor among the subtypes (Fig. 2). AF was shown in 71 (29.7\%) of 239 patients. The highest AF rate of $91 \%$ was seen in the patients with CEI (Fig. 3). Fifty-nine $(83 \%)$ of 71 patients had nonvalvular AF. AF was the highest risk factor in patients with CEI.

The outcomes in each subtype of the disease were the following: excellent/good in LI (74.4\%), 
ATI $(60.4 \%)$, CEI $(37.2 \%)$. The fatal rates were $0 \%$ in the patients with TIA and LI, 6\% in ATI, and $35.1 \%$ in CEI. CEI, followed by ATI and LI showed the worst outcome among the subtypes of the disease (Fig. 4). The better outcomes at discharge, including the patients with excellent, good outcomes, and slight and moderate disability were noted in 84

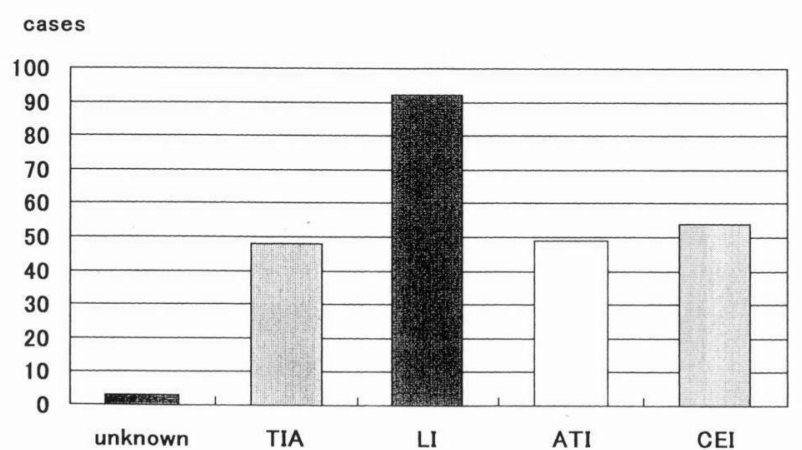

Fig. 1. The number of the patients in each subtype of the disease.

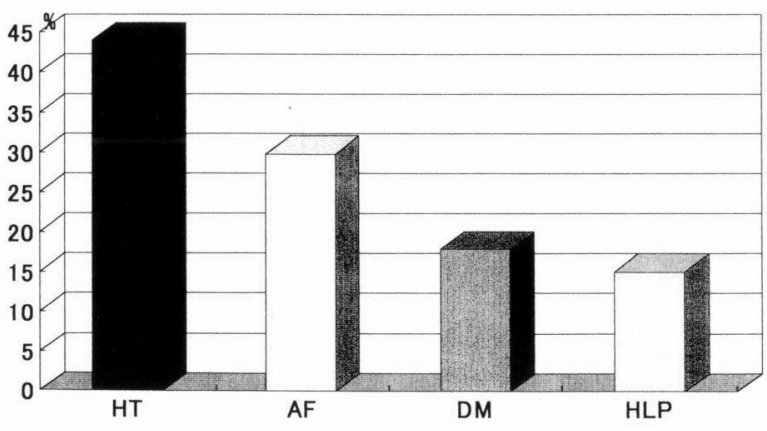

Fig. 2. Frequency of risk factors in regard to the ischemic cerebrovascular disease.

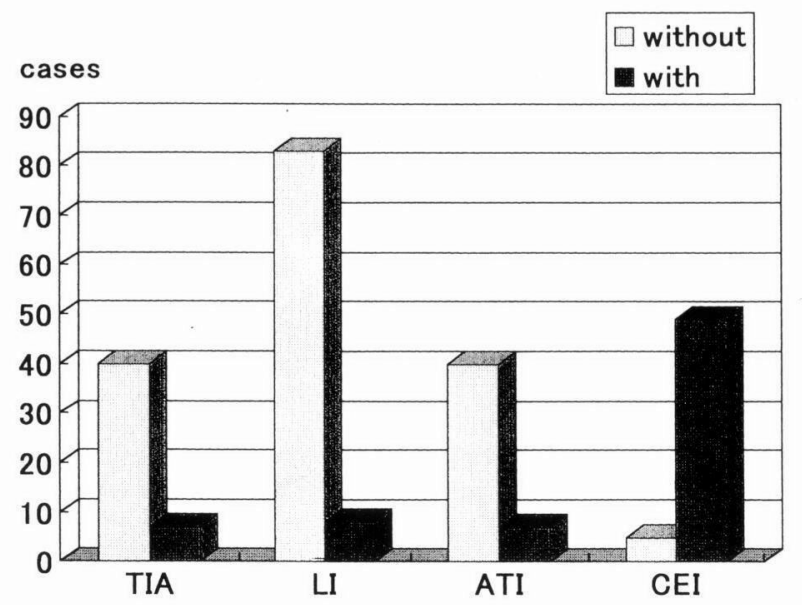

Fig. 3. Distribution of $\mathrm{AF}$ in regard to subtypes of the disease.
(93\%) of 91 patients with LI, $38(83 \%)$ of 48 patients with ATI and 26 (49\%) of 53 patients with CEI (Fig. $5)$. The worse outcomes, including the patients with severe disability, vegetative state and death were seen in $7(7 \%)$ of 91 patients with LI, $10(20.1 \%)$ of 48 patients with ATI, and 27 (50.9\%) of 53 patients with CEI (Fig. 5).

Co-relations were analyzed between the worse outcomes at discharge and the risk factors in each subtype of the disease. The statistical analysis indicated that age, gender, and AF were the determinants of the worst outcomes of the disease (Table 1). Furthermore, AF was determined to be a significant risk factor contributing to the worse outcomes among the subtypes of the disease (Table 2).

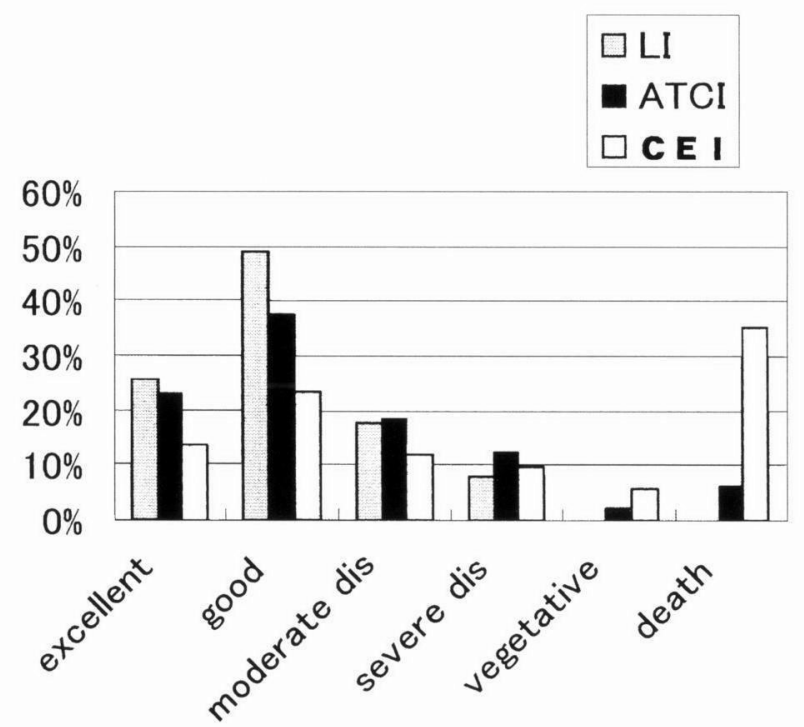

Fig. 4. Outcomes and subtypes of the disease.

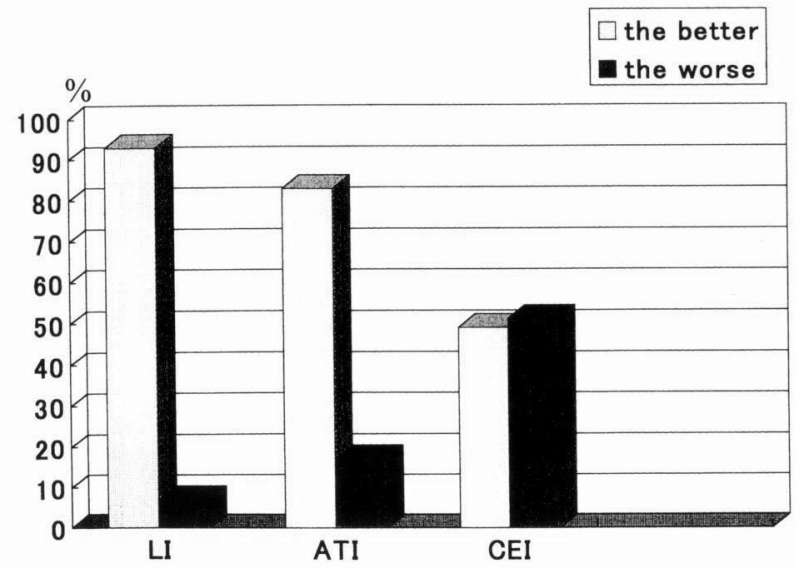

Fig. 5. The better and the worse outcomes in regard to the subtypes of the disease. 
TABLE 1.

Determinants of the worse outcome (Multiple logistic regression analysis)

\begin{tabular}{lrcc}
\hline \multicolumn{1}{c}{ Variables } & \multicolumn{1}{c}{$\beta$} & SE & Probability \\
\hline Age & 0.096 & 0.021 & $\mathrm{p}<0.001$ \\
Gender & -1.331 & 0.374 & $\mathrm{p}<0.001$ \\
Hypertension & -0.016 & 0.337 & $\mathrm{NS}$ \\
DM & -0.130 & 0.456 & $\mathrm{NS}$ \\
Hyperlipidemia & -2.153 & 1.029 & $\mathrm{p}<0.05$ \\
AF & 2.367 & 0.383 & $\mathrm{p}<0.0001$ \\
NVAF or Others & -0.591 & 0.666 & $\mathrm{NS}$ \\
\hline
\end{tabular}

DM: diabetes mellitus; AF: atrial fibrillation; NVAF: nonvalvular atrial fibrillation; $\beta$ : coefficient; SE: standard error NS: not significant

TABLE 2.

Determinants of the worse outcome (Multiple logistic regression analysis adjusted for age and gender)

\begin{tabular}{crcc}
\hline Variables & \multicolumn{1}{c}{$\beta$} & SE & Probability \\
\hline AF & 2.325 & 0.423 & 0.00001 \\
HLP & -1.868 & 1.055 & NS \\
\hline
\end{tabular}

AF: atrial fibrillation; HLP: hyperlipidemia; $\beta$ : coefficient; SE: standard error; NS: not significant

\section{DISCUSSION}

Increasing population in the geriatric with female dominancy has been indicated in Japan, and the increased rate of AF is reported to be in parallel with the age $[8,9]$. But, in the present study, it is clearly identified that AF is an independent risk factor associated with the worse outcomes of the subtypes of the disease. Our study suggested that AF might play a significant role in association with the causal mechanism of LI and ATCI. In their study, Moulin documented that embolic heart disease and large-artery disease were the main cause of small deep infarct of their patients and that LI may be caused not only by intracranial hypertensive arteriolopathy, but also by cardiac embolism [10]. In the present study, CEI presented the worst outcome among the subtypes of the disease and furthermore, statistical analysis indicated that AF was the most significant risk factor associated with the outcomes of the ischemic cerebrovascular disease. High recurrence rate in the early stage of the onset and multiple, and the presence of large cerebral infarction in the patients with CEI are reported and this may be related to the worse outcomes [1,11-13].
Further study is needed to elucidate the causal mechanism between cerebral infarction and AF to provide an appropriate treatment options for the patients with ischemic cerebrovascular disease and to achieve much better outcomes.

\section{CONCLUSION}

Present study showed that AF was a significant risk factor contributing to the worse outcomes in patients with ischemic cerebrovascular disease.

\section{REFERENCES}

1. Cerebral Embolism Task Force. Cardiogenic brain embolism. Arch Neurol 1986; 43:71-84.

2. Davis PH, Dambrosa JM, Schoenberg BS, Schoenberg DG, Pritchard DA et al. Risk factors for ischemic stroke: A prospective study in Rochester, Minnesota. Ann Neurol 1987; 22:319-327.

3. Colandrea MA, Friedman GD, Nichaman MZ, and Lynd CN. Systolic hypertension in the elderly. Circulation 1970; 41:239-245.

4. Rossner S, Kjellin KG, Mettinger KL, Siden A, and Soderstrom CE. Normal serum-cholesterol but low H.D.L.-cholesterol concentration in young patients with ischemic cerebrovascular disease. Lancet 1978; 1:577. 579.

5. Kannel WB, and McGee DL. Diabetes and cardiovascular disease. JAMA 1979; 241:2035-2038.

6. WHO MONICA project principal investigators. The world health organization MONICA project (monitoring trends and determinants in cardiovascular disease): A major international collaboration. J Clin Epidemiol 1988; 41:105-114.

7. Jennet B, and Bond M. Assessment of outcome after severe brain damage: A practical scale. Lancet 1975; $1: 480-484$.

8. Kiyohara Y, Ueda K, Hasuo J, Wada J, Kawano H et al. Type-specific incidence of cerebral infarction and its changing pattern during the long-term prospective population survey in the Hisayama town, Japan. Stroke 21 (Suppl 1) 1990; 35.

9. Wolf PA, Abott RD, and Kannel WB. Atrial fibrillation: A major contributor to stroke in the elderly. The Framingam study. Arch Intern Med 1987; 147:1561-1564.

10. Moulin T, Tatu L, Vuillier F, Berger E, Chavot D et al. Role of a stroke data bank in evaluating cerebral infarction subtypes: Patterns and outcome of 1776 consecutive patients from the Besancon stroke registry. Cerebrovasc Dis 2000; 10:261-271.

11. Cerebral Embolism Study Group. Immediate anticoagulation of embolic stroke: A randomized trial. Stroke 1983; 14:668-676.

12. Cerebral Embolism Task Force. Cardiogenic brain embolism: The second report of the cerebral embolism task force. Arch Neurol 1989; 46:727-743. 
13. Yamanouchi $\mathrm{H}$, Tomonaga $\mathrm{M}$, Shimada $\mathrm{H}$, Matsushita $\mathrm{S}$, Kuramoto $\mathrm{K}$ et al. Nonvalvular atrial fibrillation as a cause of fatal massive cerebral infarction in the elderly. Stroke 1989; 20:1653-1656. 